\title{
Reactor mixing angle from hybrid neutrino masses
}

\section{Aristizabal Sierra ${ }^{a}$ and I. de Medeiros Varzielas ${ }^{b}$}

\author{
${ }^{a}$ IFPA, Dep. AGO, Universite de Liege, \\ Bat B5, Sart Tilman B-4000 Liege 1, Belgium \\ ${ }^{b}$ Department of Physics, University of Basel, \\ Klingelbergstr. 82, CH-4056 Basel, Switzerland \\ E-mail: daristizabal@ulg.ac.be, ivo.de@unibas.ch
}

ABSTRACT: In terms of its eigenvector decomposition, the neutrino mass matrix (in the basis where the charged lepton mass matrix is diagonal) can be understood as originating from a tribimaximal dominant structure with small deviations, as demanded by data. If neutrino masses originate from at least two different mechanisms, referred to as "hybrid neutrino masses", the experimentally observed structure naturally emerges provided one mechanism accounts for the dominant tribimaximal structure while the other is responsible for the deviations. We demonstrate the feasibility of this picture in a fairly model-independent way by using lepton-number-violating effective operators, whose structure we assume becomes dictated by an underlying $A_{4}$ flavor symmetry. We show that if a second mechanism is at work, the requirement of generating a reactor angle within its experimental range always fixes the solar and atmospheric angles in agreement with data, in contrast to the case where the deviations are induced by next-to-leading order effective operators. We prove this idea is viable by constructing an $A_{4}$-based ultraviolet completion, where the dominant tribimaximal structure arises from the type-I seesaw while the subleading contribution is determined by either type-II or type-III seesaw driven by a non-trivial $A_{4}$ singlet (minimal hybrid model). After finding general criteria, we identify all the $\mathbb{Z}_{N}$ symmetries capable of producing such $A_{4}$-based minimal hybrid models.

Keywords: Beyond Standard Model, Neutrino Physics, Discrete and Finite Symmetries ARXIV EPRINT: 1404.2529 


\section{Contents}

1 Introduction 1

2 Perturbations of the TBM neutrino mass matrix 2

2.1 Sum-rules and constraints on neutrino observables 7

3 Renormalizable $A_{4}$ hybrid seesaw $r$

3.1 The type-II seesaw case 11

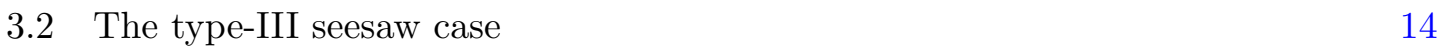

4 Conclusions $\quad 15$

\section{Introduction}

With the advent of neutrino oscillation data, in particular in the early 2000's, the tribimaximal (TBM) neutrino mixing pattern [1] became a paradigm for model building aiming to understand the neutrino mixing through an underlying flavor symmetry. The paradigm however, has been challenged by recent reactor experiments $[2,3]$ which have excluded a zero reactor mixing angle at more than $10 \sigma$ [4].

Expanding the neutrino mass matrix in terms of its eigenvectors, and with current neutrino data taken into account [4-6], one can quantify in a useful way the deviations in the structure of the mass matrix from the TBM-form [7], ${ }^{1}$ are then small due to the "small" measured value of the reactor mixing angle $\theta_{13}$. Given that the observations are consistent with an approximate TBM-form, the idea of an underlying flavor symmetry responsible for the neutrino mixing pattern is arguably well motivated. Indeed a very widely explored possibility is that of a flavor symmetry $G$ provides at the leading order (LO) a TBM-form neutrino mass matrix and the deviations demanded by data are obtained through next-to-leading-order (NLO) corrections. This approach however, at least in the most simple implementations, does not provide a compelling solution since quite often a non-zero reactor angle implies either a solar or atmospheric angles (or both) outside their experimental ranges [12-14].

Since the NLO approach is not particularly favored by data, it is important to consider alternative possibilities. In ref. [7], it was argued that the TBM-form plus small deviations structure, can be interpreted as a hint that different mechanisms participate in the generation of neutrino masses, so-called "hybrid neutrino masses". The idea is that of

\footnotetext{
${ }^{1}$ As far as we know, this type of eigenvector decomposition was first used in the context of a neutrino mass matrix with exact TBM-form to show the viability of leptogenesis in models exhibiting interplay between type-I and type-II seesaw [8], something which at leading order (LO) in the effective operator tower is not possible within the type-I seesaw [9-11].
} 
starting with a Lagrangian invariant under $G$, in which after $G$ and electroweak symmetry breaking one mechanism accounts for the TBM-form while the deviations are provided by the other mechanism (rather than by NLO corrections), with both contributions to the neutrino mass matrix entering at the same order, generically LO. This type of scenario was investigated before in the context of bi-maximal mixing [15] and also in the case of TBM [16]. In [17] it was explored in models with extra dimensions. More recently in [1820], type-I seesaw [21-28] was assumed to be responsible for the leading TBM-form and type-II seesaw [29-32] contributions introduced the deviations to TBM.

Here we intend to show the feasibility of the whole idea under fairly general assumptions, in the sense that rather than assuming specific neutrino mass generating mechanisms we deal with a discussion based on lepton-number-violating effective operators. We identify the minimal $A_{4}$ representations which render this idea plausible by assuming that the lepton sector obeys an $A_{4}$ flavor symmetry similar to the Altarelli-Feruglio (AF) model [33], where the charged lepton Yukawa couplings hierarchies are dictated by a Froggatt-Nielsen (FN) mechanism [34]. We demonstrate that within this context, if the non-zero reactor angle arises from "hybrid neutrino masses" the solar and the atmospheric angles are always within their experimental ranges, in contrast to the "standard" procedure involving NLO effective operators where - in general - this cannot be guaranteed. More specifically, and in order to illustrate the general picture, we construct explicitly $A_{4}$-based ultraviolet (UV) completions akin to [35], with the leading TBM-form originating from type-I seesaw - but importantly the deviations arise from type-II or type-III [36]. In addition to this and being UV complete with explicit messenger fields, our construction further differs from that in [18]: rather than a flavon $A_{4}$ triplet with a certain assumed vacuum expectation value (vev), we use a non-trivial $A_{4}$ singlet with a corresponding vev consistently explained by the alignment sector.

The rest of this letter is organized as follows. In section 2, by using effective LO lepton-number-breaking operators, we study the possible deviations which can emerge in $A_{4}$-inspired frameworks. Section 3 discusses a viable UV completion based on type-I plus either type-II or type-III seesaw. Finally, in section 4 we summarize and present our conclusions.

\section{Perturbations of the TBM neutrino mass matrix}

If neutrino masses are generated by two different mechanisms, with one accounting for the TBM structure of the neutrino mass matrix and the other for the deviations required by experimental data, the full neutrino mass matrix can be written according to

$$
m_{\nu}=m_{\nu}^{\mathrm{TBM}}+\delta m_{\nu}
$$

with the leading contribution $m_{\nu}^{\mathrm{TBM}}$ being determined by

$$
m_{\nu}^{\mathrm{TBM}}=U_{\mathrm{TBM}} \cdot \hat{m}_{\nu} \cdot U_{\mathrm{TBM}}^{T},
$$

where $U_{\text {TBM }}$ corresponds to the leptonic mixing matrix with mixing angles fixed according to their TBM values $\left(\sin \theta_{23}=1 / \sqrt{2}, \sin \theta_{12}=1 / \sqrt{3}\right.$ and $\left.\sin \theta_{13}=0\right)$. The perturbation 


\begin{tabular}{|c|cccc|cc|ccccc|ccc|}
\hline Fields & $L$ & $e^{c}$ & $\mu^{c}$ & $\tau^{c}$ & $H_{d}$ & $H_{u}$ & $\theta$ & $\phi_{l}$ & $\phi_{\nu}$ & $\xi$ & $\tilde{\xi}$ & $\phi_{l}^{0}$ & $\phi_{\nu}^{0}$ & $\xi^{0}$ \\
\hline$A_{4}$ & $\mathbf{3}$ & $\mathbf{1}$ & $\mathbf{1}^{\prime \prime}$ & $\mathbf{1}^{\prime}$ & $\mathbf{1}$ & $\mathbf{1}$ & $\mathbf{1}$ & $\mathbf{3}$ & $\mathbf{3}$ & $\mathbf{1}$ & $\mathbf{1}$ & $\mathbf{3}$ & $\mathbf{3}$ & $\mathbf{1}$ \\
$\mathbb{Z}_{N}$ & 1 & -1 & -1 & -1 & 0 & $a$ & 0 & 0 & $a^{\prime}$ & $a^{\prime}$ & $a^{\prime}$ & 0 & $a^{\prime \prime}$ & $a^{\prime \prime}$ \\
$\mathrm{U}(1)_{\mathrm{FN}}$ & 0 & 2 & 1 & 0 & 0 & 0 & -1 & 0 & 0 & 0 & 0 & 0 & 0 & 0 \\
$\mathrm{U}(1)_{\mathrm{R}}$ & 1 & 1 & 1 & 1 & 0 & 0 & 0 & 0 & 0 & 0 & 0 & 2 & 2 & 2 \\
$\mathrm{U}(1)_{\mathrm{Y}}$ & $-1 / 2$ & +1 & +1 & +1 & $-1 / 2$ & $+1 / 2$ & 0 & 0 & 0 & 0 & 0 & 0 & 0 & 0 \\
\hline
\end{tabular}

Table 1. Charge assignments of the different superfields defining the AF model, which we have taken to be responsible for the LO TBM-form of the neutrino mass matrix. We have $a^{\prime} \equiv-2 a-2$ and $a^{\prime \prime} \equiv 4 a+4$ and denote $N-1$ as -1 for simplicity.

matrix $\delta m_{\nu}$ must produce a non-vanishing and sizable reactor angle while at the same time yielding not too large corrections to the other two mixing angles. At the model-independent level several conclusions can be drawn, in particular: a non-vanishing reactor angle requires the perturbation matrix to induce sizable deviations mainly in the $m_{\nu_{12}}$ and $m_{\nu_{13}}$ entries, and these deviations in turn imply departures of $\sin \theta_{23}$ and $\sin \theta_{12}$ from their TBM form, being more pronounced in the atmospheric sector [7].

In trying to keep our approach as model-independent as possible, we develop our analysis by considering first effective $\Delta L=2$ operators accounting for both $m_{\nu}^{\mathrm{TBM}}$ and $\delta m_{\nu}$. But to study more in-depth the viability of this framework where deviations from the TBM form arise from a separate mechanism, one must consider particular models where one may have the structure of $\delta m_{\nu}$ determined by the underlying flavor symmetry. Due to this, we assume a supersymmetric implementation of an $A_{4}$ lepton flavor symmetry (given its relative simplicity) [33].

In this context, getting $m_{\nu}^{\text {TBM }}$ requires, in addition to the standard model superfields transforming non-trivially under $A_{4}$, the presence of new superfields (see table 1 , where in contrast to the original model in [33] we have considered $\mathbb{Z}_{N}$ transformations and the possibility of the "up-type" Higgs transforming non-trivially under $\left.\mathbb{Z}_{N}\right) . \mathrm{U}(1)_{Y}$ denotes the hypercharge and $\mathrm{U}(1)_{R}$ the R-symmetry, The so-called alignment superfields (with $\mathrm{U}(1)_{R}$ assignment 2 , denoted by superscript 0 ) will lead to specific non-vanishing vevs for the so-called flavons (with $\mathrm{U}(1)_{R}$ assignment 0 ).

We briefly review the relevant superpotential terms. In the following, curly brackets denote the contraction of $A_{4}$ triplets into $A_{4}$ singlets, $\{A B\}=A_{1} B_{1}+A_{2} B_{3}+A_{3} B_{2}$, $\{A B\}^{\prime \prime}=A_{2} B_{2}+A_{3} B_{1}+A_{1} B_{3},\{A B\}^{\prime}=A_{3} B_{3}+A_{1} B_{2}+A_{2} B_{1}$, and similarly for the 3-triplet contractions.

The charged lepton Yukawa couplings have hierarchical masses due to the FN mechanism [34]: the $\mathrm{U}(1)_{\mathrm{FN}}$ flavor symmetry shown in table 1 is broken by $\langle\theta\rangle$

$$
W_{\ell}^{\mathrm{Eff}}=\frac{y_{e}}{\Lambda} \lambda^{2} e^{c}\left\{L \phi_{l}\right\} H_{d}+\frac{y_{\mu}}{\Lambda} \lambda \mu^{c}\left\{L \phi_{l}\right\}^{\prime} H_{d}+\frac{y_{\tau}}{\Lambda} \tau^{c}\left\{L \phi_{l}\right\}^{\prime \prime} H_{d}+\text { H.c. },
$$

with $\lambda=\langle\theta\rangle / \Lambda$ being the FN suppression factors and $\Lambda$ the FN cutoff scale. The superpotential for the neutrinos is

$$
W_{\nu}^{\mathrm{Eff}}=\frac{\left(x_{A} \xi+\tilde{x}_{A} \tilde{\xi}\right)}{\Lambda^{2}}\{L L\} H_{u} H_{u}+\frac{x_{B}}{\Lambda^{2}}\left\{\phi_{\nu} L L\right\} H_{u} H_{u} .
$$


The LO TBM comes from the flavon vevs:

$$
\left\langle\phi_{l}\right\rangle=v_{l}(1,0,0), \quad\left\langle\phi_{\nu}\right\rangle=v_{\nu}(1,1,1), \quad\langle\xi\rangle=u,
$$

provided by the alignment superpotential:

$$
\begin{aligned}
W_{\phi}= & M\left\{\phi_{l}^{0} \phi_{l}\right\}+g\left\{\phi_{l}^{0} \phi_{l} \phi_{l}\right\}+g_{1}\left\{\phi_{\nu}^{0} \phi_{\nu} \phi_{\nu}\right\}+g_{2} \tilde{\xi}\left\{\phi_{\nu}^{0} \phi_{\nu}\right\}+g_{3} \xi^{0}\left\{\phi_{\nu} \phi_{\nu}\right\} \\
& +g_{4} \xi^{0} \xi \xi+g_{5} \xi^{0} \xi \tilde{\xi}+g_{6} \xi^{0} \tilde{\xi} \tilde{\xi} .
\end{aligned}
$$

These superpotential terms are invariant under all the symmetries in table 1 . The presence of the $\mathbb{Z}_{N}$ symmetry implies that charged lepton and neutrino masses arise from two independent sets of fields, while the $\mathrm{U}(1)_{R}$ separates the superfields as those that correspond to fermions of the standard model (first four columns in table 1), alignment (or "driving") fields (last three columns in table 1), and symmetry breaking fields.

After electroweak and flavor symmetry breaking the set of interactions in (2.4), combined with the vacuum alignment in (2.5) and the appropriate $A_{4}$ group index contractions, yields for the effective neutrino mass matrix

$$
m_{\nu}^{\mathrm{TBM}}=\left(\begin{array}{ccc}
A+2 B / 3 & -B / 3 & -B / 3 \\
\cdot & 2 B / 3 & A-B / 3 \\
\cdot & \cdot & 2 B / 3
\end{array}\right),
$$

with $\left\langle H_{u}\right\rangle=v_{u}$ and the parameters in the RH neutrino mass defined as

$$
A=2 x_{A} v_{u}^{2} \frac{u}{\Lambda^{2}} \quad \text { and } \quad B=2 x_{B} v_{u}^{2} \frac{v_{\nu}}{\Lambda^{2}} .
$$

In general this structure is maintained only at LO, unless a specific UV completion guarantees the absence of NLO contributions [35]. The NLO contributions modify $m_{\nu}^{\mathrm{TBM}}$ directly through a change in the neutrino terms adding to (2.4) or indirectly by shifting the vacuum alignment in (2.5). Constraining the discussion to only this setup, this implies that $\mathcal{O}\left(\delta m_{\nu} / m_{\nu}^{\mathrm{TBM}}\right) \sim \operatorname{vev} / \Lambda \equiv \delta$. For values of $\delta$ of $\mathcal{O}(0.1)$, these corrections can account for a non-vanishing reactor angle but at the same time imply deviations of the solar and atmospheric angle from their TBM values, often outside of their experimental ranges [12-14]. For smaller $\delta$, below $\sim 0.1$, the TBM structure is less affected.

If additional $\left(A_{4}\right)$ flavons are present, they can allow additional lepton-number-breaking effective operators that deviate from $m_{\nu}^{\mathrm{TBM}}$. If the new operators are less suppressed than the the NLO operators of the "original" model, the deviations from TBM will be naturally dictated by the new contribution, which we denote as $\delta m_{\nu}^{\text {Extra }}$. This can happen if $\delta m_{\nu}^{\mathrm{NLO}}<\delta m_{\nu}^{\mathrm{Extra}} \sim \mathcal{O}(0.1)$, e.g. if the NLO operators are forbidden by the UV completion. Then $\delta m_{\nu}^{\text {Extra }}$ must generate deviations consistent with data. Given the lepton doublets transformation properties, three $A_{4}$ assignments for an extra flavon are possible: a triplet 3 [18], a singlet $\mathbf{1}^{\prime \prime}$ or a singlet $\mathbf{1}^{\prime}$. For the triplet to produce a deviation in the TBM leading structure, the flavon vev should differ from that of $\phi_{\nu}$ in $(2.5)$, otherwise $\delta m_{\nu}^{\text {Extra }}$ will just shift the parameter $B$ in (2.7), keeping its TBM-form. ${ }^{2}$ Instead, with the new

\footnotetext{
${ }^{2}$ The same will happen with the parameter $A$ if the new flavon is a trivial $A_{4}$ singlet, $\mathbf{1}$.
} 
flavon transforming as $\mathbf{1}^{\prime \prime}$ or $\mathbf{1}^{\prime}$, a non-vanishing vev will be sufficient to produce deviations from TBM, so the non-trivial singlet choices are simpler.

A minimal hybrid singlet-based AF-inspired model will then consist of the AF fields plus new flavons, namely $\xi^{\prime}$ and its corresponding "driving" field $\xi^{0^{\prime}}$ or $\xi^{\prime \prime}$ and $\xi^{0^{\prime \prime}}$. Two conditions have to be fulfilled for the emerging scheme to be phenomenologically consistent: (a) the TBM deviations induced by the new Yukawa operators arising from the presence of the singlet should be consistent with data [4-6]; (b) the new "driving sector" operators should lead to a non-vanishing singlet vev and at the same time not spoil the vev alignments (2.5) assuring the leading order TBM structure.

We consider separately the two types of minimal $A_{4}$-based hybrid models: with $\xi^{\prime \prime}$ or with $\xi^{\prime}$. The respective dimension six effective operators would be:

$$
\mathcal{O}_{6}=\frac{z}{\Lambda^{2}}\{L L\}^{\prime} \xi^{\prime \prime} H_{u} H_{u}, \quad \mathcal{O}_{6}=\frac{z}{\Lambda^{2}}\{L L\}^{\prime \prime} \xi^{\prime} H_{u} H_{u} .
$$

$\mathbb{Z}_{N}$ invariance then fixes the charge of the new flavon to match that of the AF trivial singlet flavon $\xi$. Such a charge assignment typically renders the hybrid scheme ineffective due to unavoidable additional invariants. Specifically, if some $\{X\} \xi$ coupling exists ( $X$ being a combination of fields belonging to the UV completed model, particularly $A_{4}$ invariants made of two triplets), $\mathbb{Z}_{N}$ invariance will also allow the coupling $\{X\}^{\prime} \xi^{\prime \prime}$ (or $\{X\}^{\prime \prime} \xi^{\prime}$ ). In other words, the $\mathbb{Z}_{N}$ equality implies that both operators, the one yielding TBM and the one responsible for the deviations, will stem from the same (UV complete) mechanism. This is certainly the case in the AF-inspired models, where equal $\mathbb{Z}_{N}$ charges lead the $\xi$ and $\xi^{\prime \prime}$ (or $\xi^{\prime}$ ) to contribute through type-I seesaw. The conclusion is more general though: minimal hybridization will often require a distinct charge under some Abelian symmetry, even though the assignments under the non-Abelian symmetry are already distinct. We will refer to this as the hybridization statement.

There is another argument supporting an even stronger hybridization statement, and it has to do with the hybridization condition (b). In a specific UV completion of the AF-inspired models, a mismatch between $\xi$ and $\xi^{\prime \prime}$ (or $\xi^{\prime}$ ) charges can be given by a sign mismatch (where a charge of $-a$ corresponds to $N-a$ under $\mathbb{Z}_{N}$ ). Such a mismatch avoids the coupling $\{X\}^{\prime} \xi^{\prime \prime}$ (or $\{X\}^{\prime \prime} \xi^{\prime}$ ). However, new terms at the "driving sector" level will be allowed, namely

$$
W_{\phi}^{\text {New }} \supset \xi^{\prime \prime}\left\{\phi_{l}^{0} \phi_{\nu}\right\}^{\prime}+\xi^{\prime \prime}\left\{\phi_{\nu}^{0} \phi_{l}\right\}^{\prime}
$$

or the equivalent terms for $\xi^{\prime}$. The first term destroys the $\left\langle\phi_{l}\right\rangle$ alignment in (2.5), while the second term spoils the $\left\langle\phi_{\nu}\right\rangle$ alignment, and therefore any of them harm the LO TBM structure. A mismatch beyond just a sign flip is thus mandatory in the AF-inspired models. This constraint applies in general to models where some flavons or alignment fields are neutral under the Abelian symmetries.

At the effective level, such a mismatch is possible only if $\xi$ and $\xi^{\prime \prime}$ (or $\xi^{\prime}$ ) participate in operators of different dimensionality. If one aims to argue that the hybrid structure accounts for the small TBM deviations it is desirable to have the deviations appear at 

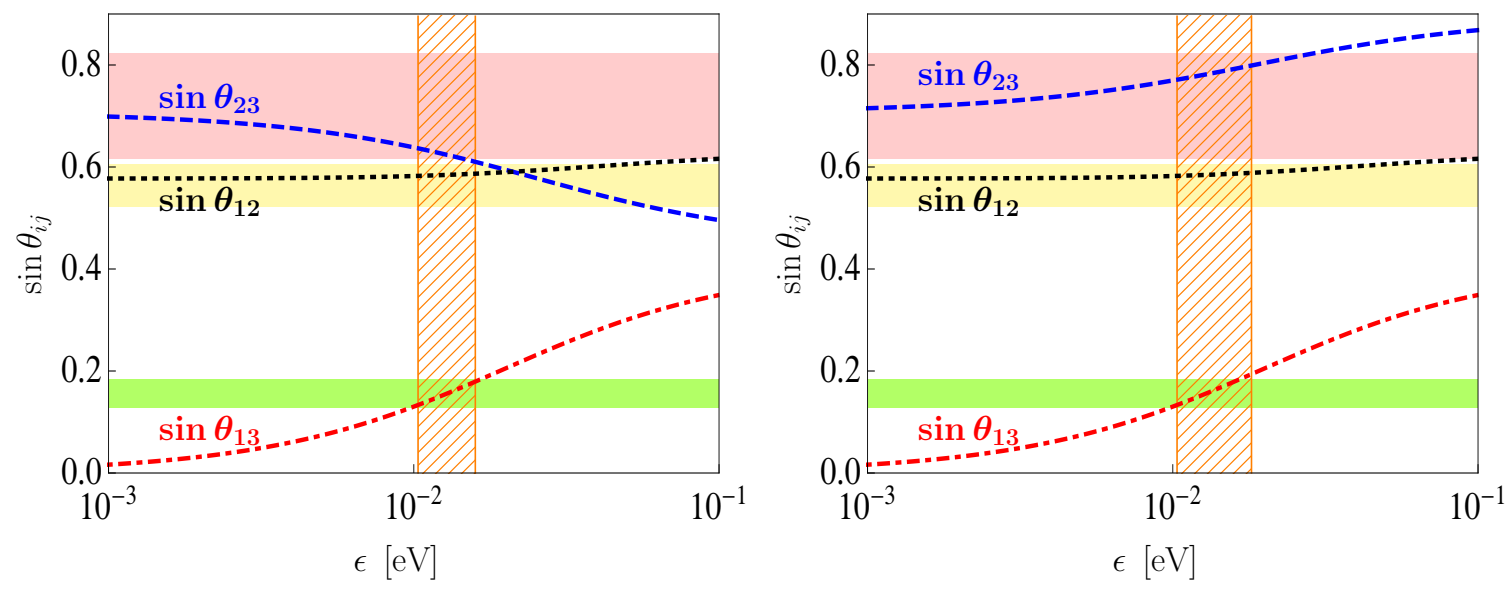

Figure 1. Deviations on the different mixing angles induced by the perturbation matrices $\delta m_{12}^{33}$ (left plot) and $\delta m_{13}^{22}$ (right plot). The horizontal stripes indicate the $3 \sigma$ allowed range for the atmospheric angle (top stripe), solar angle (middle stripe) and reactor angle (bottom stripe). The hatched vertical stripe indicates the range that parameter $\epsilon$ should have in order for the deviations to be consistent with current neutrino data [4-6].

higher order, the simplest cases are then

$$
\begin{aligned}
& \mathcal{O}_{7}=\frac{z}{\Lambda^{3}}\{L L\}^{\prime} \xi^{\prime} \xi^{\prime} H_{u} H_{u} \quad \stackrel{\text { After SB }}{\longrightarrow} \quad \delta m_{\nu}=\delta m_{12}^{33}=\epsilon\left(\begin{array}{lll}
0 & 1 & 0 \\
1 & 0 & 0 \\
0 & 0 & 1
\end{array}\right), \\
& \mathcal{O}_{7}=\frac{z}{\Lambda^{3}}\{L L\}^{\prime \prime} \xi^{\prime \prime} \xi^{\prime \prime} H_{u} H_{u} \quad \stackrel{\text { After SB }}{\longrightarrow} \quad \delta m_{\nu}=\delta m_{13}^{22}=\epsilon\left(\begin{array}{lll}
0 & 0 & 1 \\
0 & 1 & 0 \\
1 & 0 & 0
\end{array}\right),
\end{aligned}
$$

where we have defined $\epsilon \equiv z v_{u}^{2} \bar{u}^{2} / \Lambda^{3}$ and have assumed that alignment superpotential leads to $\left\langle\xi^{\prime \prime}\left({ }^{\prime}\right)\right\rangle=\bar{u} \neq 0$. Note that with the deviations dictated by $\mathcal{O}_{7}$ the $\mathbb{Z}_{N}$ charge of the non-trivial singlet flavon is $-1-a$, half the charge of $\xi$.

In summary, if the leading TBM-form of the neutrino mass matrix arises from this type of $A_{4}$ setup, the simplest lepton-number-breaking operators which can induce deviations from the TBM-form are determined by the presence of a flavon transforming as a nontrivial $A_{4}$ singlet [14] (see also [37-39]). Thus, within this context, two minimal setups can be defined, namely

$$
\begin{array}{rlr}
A_{4}-\Xi^{\prime \prime} \text { model : } & m_{\nu}=m_{\nu}^{\mathrm{TBM}}+\delta m_{12}^{33} \quad \text { with } \Xi^{\prime \prime} \equiv \xi^{\prime} \xi^{\prime}, \\
A_{4}-\Xi^{\prime} \text { model : } & m_{\nu}=m_{\nu}^{\mathrm{TBM}}+\delta m_{13}^{22} \quad \text { with } \Xi^{\prime} \equiv \xi^{\prime \prime} \xi^{\prime \prime} .
\end{array}
$$

Assuming $v_{\nu} \sim u$ and $x_{A} \sim x_{B}$, the relative size between the two terms becomes determined by $\delta m_{\nu}^{\text {Extra }} / m_{\nu}^{\mathrm{TBM}}=z \bar{u}^{2} / 2 x_{A} u \Lambda$, with the precise numerical ranges fixed by the requirement of having a mixing pattern compatible with neutrino oscillation data [4-6]. 


\begin{tabular}{|c|c|c|c|c|c|c|c|c|}
\hline OBS. & \multicolumn{2}{|c|}{$\left|m_{\text {Light }}\right|[\mathrm{eV}]$} & \multicolumn{2}{c|}{$\sum m_{\nu_{i}}[\mathrm{eV}]$} & \multicolumn{2}{c|}{$m_{\beta}[\mathrm{eV}]$} & \multicolumn{2}{c|}{$\left\langle m_{e e}\right\rangle[\mathrm{eV}]$} \\
\hline SPECT. & NO $\left(\left|m_{1}\right|\right)$ & $\mathrm{NO}\left(\left|m_{3}\right|\right)$ & NO & IO & NO & IO & NO & IO \\
\hline$\varepsilon=0$ & $\gtrsim 0.0014$ & $\bullet$ & $\gtrsim 0.078$ & $\bullet$ & $\gtrsim 0.015$ & $\bullet$ & $\gtrsim 0.0031$ & $\bullet$ \\
\hline$\varepsilon=0.1$ & $\gtrsim 0.012$ & $\gtrsim 0.062$ & $\gtrsim 0.074$ & $\gtrsim 0.22$ & $\gtrsim 0.014$ & $\gtrsim 0.077$ & $\gtrsim 0.0029$ & $\gtrsim 0.054$ \\
\hline$\varepsilon=0.2$ & $\gtrsim 0.0098$ & $\gtrsim 0.034$ & $\gtrsim 0.070$ & $\gtrsim 0.15$ & $\gtrsim 0.012$ & $\gtrsim 0.057$ & $\gtrsim 0.0023$ & $\gtrsim 0.054$ \\
\hline$\varepsilon=0.3$ & $\gtrsim 0.0076$ & $\gtrsim 0.019$ & $\gtrsim 0.065$ & $\gtrsim 0.12$ & $\gtrsim 0.010$ & $\gtrsim 0.049$ & $\gtrsim 0.0013$ & $\gtrsim 0.047$ \\
\hline
\end{tabular}

Table 2. Lower limits on neutrino observables for the normal (NO) and inverted (IO) ordering of the masses. The dot indicates that the IO is not allowed in the exact TBM limit. The results have been taken from [40].

Taking $m_{\nu}^{\mathrm{TBM}}$ as given in (2.2), and fixing the lightest light neutrino mass to be somewhere below $10^{-2} \mathrm{eV}$ the full neutrino mass matrix becomes a function of a single parameter, $\epsilon$, i.e. $m_{\nu}=m_{\nu}(\epsilon)$. Thus, in order to check for the viability of the resulting models, one can diagonalize $m_{\nu}(\epsilon)$ and see whether for the $\epsilon$ range where $\sin \theta_{13}$ falls into its measured value the remaining two mixing angles are also within their experimental ranges. Figure 1 shows the results in both cases for the normal mass spectrum, left plot for the $A_{4}-\Xi^{\prime \prime}$ model while the right plot for the $A_{4}-\Xi^{\prime}$ model. We fixed $m_{\nu_{1}}$ according to $10^{-3} \mathrm{eV}$. These results prove that as long as $\epsilon \subset[1.2,2.2] \times 10^{-2} \mathrm{eV}$, which implies $\delta m_{\nu}^{\text {Extra }} / m_{\nu}^{\mathrm{TBM}} \sim \epsilon / m_{\nu_{3}} \sim 0.4$, one is always able to obtain a mixing pattern consistent with data. More importantly, for the range where the reactor angle falls in its $3 \sigma$ experimental range, the atmospheric and solar angles are also in the $3 \sigma$ range. This follows directly from implementing hybrid seesaw in the particular case of $A_{4}$ with non-trivial singlets, and compares favorably with the AF model, where NLO contributions required to match the observed reactor angle do not necessarily imply that the solar and atmospheric angles are in agreement with data $[12,13]$ (this is also the case for UV completions [14]).

\subsection{Sum-rules and constraints on neutrino observables}

The complex eigenvalues of the leading order TBM mass matrix in (2.7) are given by $A$, $B-A$ and $B+A$. They can be identified with the TBM eigenvectors $\boldsymbol{v}_{\mathbf{1}}=(-2,1,1) / \sqrt{6}$, $\boldsymbol{v}_{\mathbf{2}}=(1,1,1) / \sqrt{3}$, and $\boldsymbol{v}_{\mathbf{3}}=(0,-1,1) / \sqrt{2}$, thus implying:

$$
m_{\nu_{1}}=A+B, \quad m_{\nu_{2}}=A, \quad m_{\nu_{3}}=B-A .
$$

Therefore, at the TBM leading order level neutrino masses obey the following sum-rule:

$$
m_{\nu_{1}}=2 m_{\nu_{2}}+m_{\nu_{3}}
$$

As has been discussed at length in [40], neutrino mass sum-rules add further constraints on neutrino observables: the lightest neutrino mass $\left(m_{\text {light }}\right)$, the sum of absolute neutrino masses $\left(\sum m_{\nu_{i}}\right)$, the kinematic electron neutrino mass in $\beta$ decay $\left(m_{\beta}\right)$ and the effective mass for $0 \nu \beta \beta\left(\left\langle m_{e e}\right\rangle\right)$. In the presence of deviations from the TBM structure the sumrules and corresponding constraints change. For deviations given by (2.11) and (2.12), the 
eigenvalues — at order $\epsilon$ - are instead given by

$$
m_{\nu_{1}}=A+B-\frac{\epsilon}{2}, \quad m_{\nu_{3}}=A+\epsilon, \quad m_{\nu_{3}}=B-A+\frac{\epsilon}{2},
$$

which in turn imply the following modified sum-rule

$$
2 m_{\nu_{2}}+m_{\nu_{3}}-m_{\nu_{1}}=3 \epsilon .
$$

In [40] this type of deviation from the sum rule was parametrized as

$$
2 m_{\nu_{2}}+m_{\nu_{3}}-m_{\nu_{1}}=\varepsilon\left|m_{\text {heaviest }}^{0}\right| e^{i \phi_{3}} .
$$

This means that the results in [40] can be directly translated to our case through the equality $\varepsilon=3|\epsilon| /\left|m_{\text {heaviest }}^{0}\right|$. With $\mathcal{O}(\epsilon) \sim 0.01 \mathrm{eV}$, as required by neutrino mixing data (see figure 1, hatched (orange) vertical stripe), and fixing the heaviest neutrino mass to be $\mathcal{O}\left(\left|m_{\text {heaviest }}\right|\right) \sim 0.1 \mathrm{eV}$ one can estimate $\varepsilon$ to be order $0.1-0.3$. Thus, once the hybrid contribution is added the lower limits on the neutrino observables will obey the constraints found in [40] for $\varepsilon=0.1-0.3$, which — for completeness - we summarize in table 2. Note that in the exact TBM limit the inverted spectrum is not allowed, but becomes possible once the deviations are added.

\section{Renormalizable $A_{4}$ hybrid seesaw}

Given the viability of generating a non-vanishing reactor mixing angle from two different sets of $\Delta L=2$ effective operators, it is interesting to discuss UV complete realizations. For that aim we will have type-I seesaw responsible for the leading TBM-form while $\delta m_{\nu}$ arises from type-II (assumed from now up to section 3.2) or type-III seesaw (assumed in section 3.2). We will specialize to the case of the $A_{4}-\Xi^{\prime}$ model, results for the $A_{4}-\Xi^{\prime \prime}$ model follow directly from what will be discussed below.

Getting $m_{\nu}^{\mathrm{TBM}}$ from type-I seesaw requires the presence of $\mathrm{RH}$ neutrinos superfields $\left(\nu^{c}\right)$, which we take to transform as $A_{4}$ triplets, 3. The seesaw Yukawa couplings $L \nu^{c} H_{u}$ require the $\mathrm{RH}$ neutrinos to have a $\mathbb{Z}_{N}$ charge equal to $-1-a$, and this requirement in turn implies that the neutral sector flavons $\phi_{\nu}, \xi$ and $\tilde{\xi}$ should have $\mathbb{Z}_{N}$ charges equal to $2+2 a$, rather than as $-2-2 a$ as they do when effective operators are used instead. With $\phi_{\nu}, \xi$ and $\tilde{\xi}$ having this charge, the scalar potential terms in (2.6) require the neutral sector driving fields to have charges equal to $-4-4 a$. All in all, the introduction of the type-I seesaw changes the transformation properties of the neutral sector flavon fields in table 1 , while leaving the charged lepton sector fields unchanged.

Since we are considering a UV completion, we have only renormalizable interactions, the charged lepton superpotential in (2.3) will be obtained through messenger superfields transforming non-trivially under $\mathbb{Z}_{N}$ and $\mathrm{U}(1)_{Y}$ (see table 3 ). Apart from the changes to $\mathbb{Z}_{N}$ (matching what is required due to the neutrino sector), these are the same messengers that were employed originally in [35], meaning we also adopt the convention of coupling the left-handed $(\mathrm{RH})$ leptons to $H_{d}(\theta)$, in which case the explicit renormalizable superpotential 


\begin{tabular}{|c|cccc|cccc|}
\hline Fields & $\chi_{\tau}$ & $\chi_{1}$ & $\chi_{2}$ & $\chi_{3}$ & $\chi_{\tau}^{c}$ & $\chi_{1}^{c}$ & $\chi_{2}^{c}$ & $\chi_{3}^{c}$ \\
\hline$A_{4}$ & $\mathbf{3}$ & $\mathbf{1}^{\prime}$ & $\mathbf{1}$ & $\mathbf{1}$ & $\mathbf{3}$ & $\mathbf{1}^{\prime \prime}$ & $\mathbf{1}$ & $\mathbf{1}$ \\
$\mathbb{Z}_{N}$ & 1 & 1 & 1 & 1 & -1 & -1 & -1 & -1 \\
$\mathrm{U}(1)_{\mathrm{FN}}$ & 0 & 0 & 0 & -1 & 0 & 0 & 0 & +1 \\
$\mathrm{U}(1)_{\mathrm{R}}$ & 1 & 1 & 1 & 1 & 1 & 1 & 1 & 1 \\
$\mathrm{U}(1)_{\mathrm{Y}}$ & -1 & -1 & -1 & -1 & +1 & +1 & +1 & +1 \\
\hline
\end{tabular}

Table 3. Messenger sector for the charged lepton sector. The $\chi$ fields are the FN messenger superfields responsible for the charged lepton Yukawa couplings hierarchies.
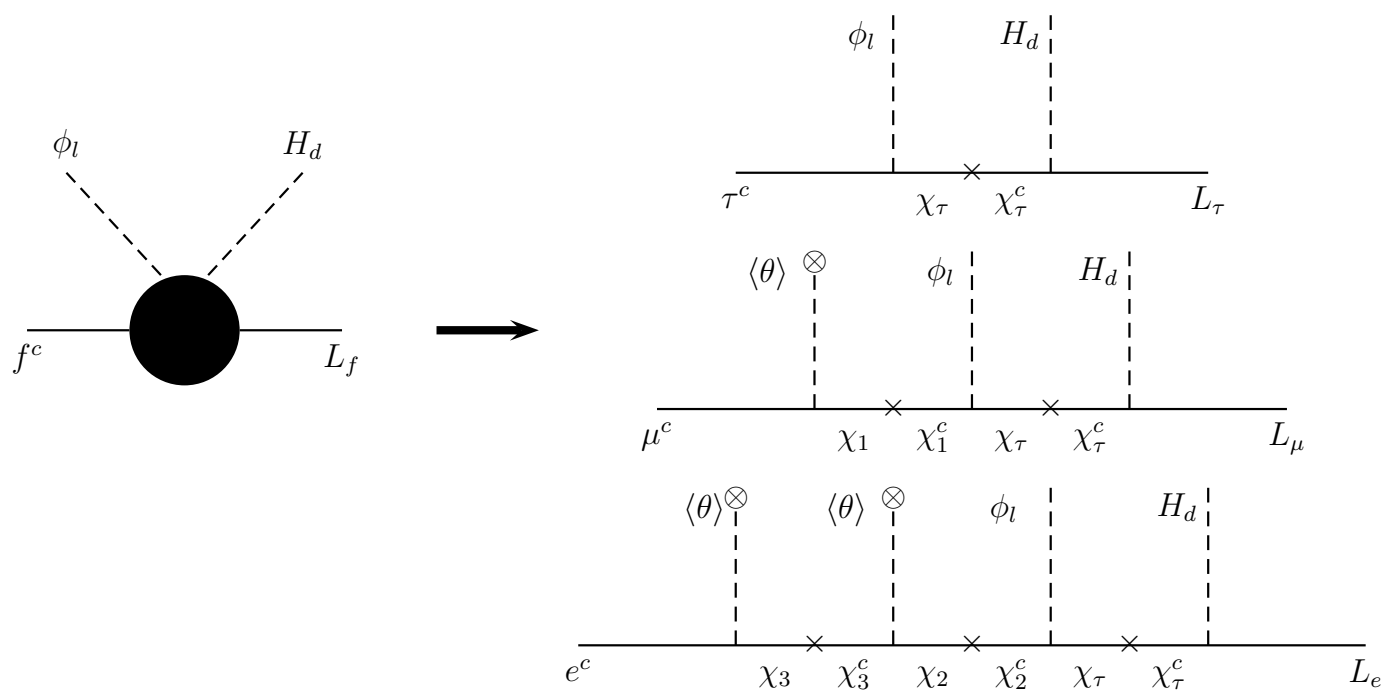

Figure 2. Effective charged lepton Yukawa operators arising from integrating out the heavy vectorlike fields $\chi_{A}$ (messenger fields) entering in the set of renormalizable interactions in the superpotential in (3.1).

reads [35]:

$$
\begin{aligned}
W_{\ell}= & y_{L \tau}\left\{L \chi_{\tau}^{c}\right\} H_{d}+y_{\tau \tau} \tau^{c}\left\{\chi_{\tau} \phi_{l}\right\}+y_{1 \tau} \chi_{1}^{c}\left\{\chi_{\tau} \phi_{l}\right\}^{\prime}+y_{\mu 1} \mu^{c} \chi_{1} \theta+y_{2 \tau} \chi_{2}^{c}\left\{\chi_{\tau} \phi_{l}\right\} \\
& +y_{32} \chi_{3}^{c} \chi_{2} \theta+y_{e 3} e^{c} \chi_{3} \theta+M_{\chi_{A}} \chi_{A} \chi_{A}+\text { H.c. },
\end{aligned}
$$

with the different couplings $y_{i j}$ being order-one numbers and the $A$ subscript denoting the different messengers in their respective mass terms. Integrating out the messengers yields the effective superpotential in (2.3), as shown in figure 2 .

The neutral renormalizable superpotential consist of two pieces, one accounting for the type-I seesaw interactions and a second piece responsible for the other sector:

$$
W_{\nu}=W_{\nu}^{(I)}+W_{\nu}^{(I I)} .
$$

With the $\mathbb{Z}_{N}$ assignments, the type-I seesaw superpotential can then be written as

$$
W_{\nu}^{(I)}=y_{N}\left\{L \nu^{c}\right\} H_{u}+\left(x_{A} \xi+\tilde{x}_{A} \tilde{\xi}\right)\left\{\nu^{c} \nu^{c}\right\}+x_{B}\left\{\phi_{\nu} \nu^{c} \nu^{c}\right\},
$$


with the corresponding alignment terms ensuring the appropriate flavon vevs given in (2.6). After flavor and electroweak symmetry breaking, the Dirac mass matrix and RH neutrino mass matrix combine through type-I seesaw into a TBM-form for the effective neutrino mass matrix, $m_{\nu}^{\mathrm{TBM}}=m_{D}\left(M_{N}\right)^{-1} m_{D}^{T}$.

Before specifying the type-II sector we will discuss some generalities regarding the flavon field $\xi^{\prime}$. As has been pointed out in section 2 , its $\mathbb{Z}_{N}$ charge is fixed to be $-1-a$ in the effective approach. As we will show, through a specific UV completion there is also the possibility to allow the charge to be the opposite choice, so we have two choices: $\pm(1+a)$. Depending on the $a$ charges and the cyclic group order $N$, the presence of $\xi^{\prime}$ and the corresponding "driving" flavon $\xi^{0^{\prime}}$ might allow the construction of the following relevant "driving sector" operators:

$$
W_{\phi}^{\mathrm{New}}=\bar{g}_{1} \xi^{\prime}\left\{\phi_{l}^{0} \phi_{l}\right\}^{\prime \prime}+\bar{g}_{2} \xi^{\prime}\left\{\phi_{\nu}^{0} \phi_{l}\right\}^{\prime \prime}+\bar{g}_{3} \xi^{\prime}\left\{\phi_{l}^{0} \phi_{\nu}\right\}^{\prime \prime}+\bar{g}_{4} \xi^{0^{\prime}}\left\{\phi_{l} \phi_{\nu}\right\}^{\prime \prime}+\bar{g}_{5} \xi^{0^{\prime}} \xi^{\prime} \xi^{\prime} .
$$

Regardless of the choice the first three operators should be forbidden otherwise the LO TBM structure dictated by the type-I seesaw sector will be spoiled. The last two terms instead will be the ones accounting for a non-vanishing $\left\langle\xi^{\prime}\right\rangle$, and so their presence is desirable. Since the positive charge choice, $1+a$, for $\xi^{\prime}$ automatically guarantees the presence of both, provided the driving field $\xi^{0^{\prime}}$ charge is $-2(1+a)$, we regard this charge assignment as the most compelling one. Note that by doing this there will be some subtlety in determining the correct $A_{4}$ contraction of the $L$, which determine the shape of the mass matrix contribution and whether the respective model is a $\Xi^{\prime}$ or a $\Xi^{\prime \prime}$ model.

The allowed $a$ and $N$ choices, i.e. the choices for which the first three terms in (3.4) are forbidden can be determined in complete generality by considering their $\mathbb{Z}_{N}$ transformation properties. Taking $\eta_{N} \equiv e^{i 2 \pi / N}$, the dangerous operators in (3.4) transform according to

$$
\begin{aligned}
& \xi^{\prime}\left\{\phi_{l}^{0} \phi_{l}\right\}^{\prime \prime} \rightarrow \eta_{N}^{(1+a)} \xi^{\prime}\left\{\phi_{l}^{0} \phi_{l}\right\}^{\prime \prime}, \\
& \xi^{\prime}\left\{\phi_{\nu}^{0} \phi_{l}\right\}^{\prime \prime} \rightarrow \eta_{N}^{-3(1+a)} \xi^{\prime}\left\{\phi_{\nu}^{0} \phi_{l}\right\}^{\prime \prime}, \\
& \xi^{\prime}\left\{\phi_{l}^{0} \phi_{\nu}\right\}^{\prime \prime} \rightarrow \eta_{N}^{3(1+a)} \xi^{\prime}\left\{\phi_{l}^{0} \phi_{\nu}\right\}^{\prime \prime} .
\end{aligned}
$$

Thus, these terms will be allowed provided the following conditions hold

$$
1+a=N \alpha, \quad-3(1+a)=N \beta, \quad 3(1+a)=N \gamma,
$$

with $\alpha, \beta, \gamma$ integers. Solutions to these equations provide constraints between the $a$ charge and the cyclic group order which when satisfied lead to inviable models, models where the LO TBM structure does not hold due to hybridization, the remaining choices are those for which an AF-inspired hybrid model becomes possible. For $\beta \neq 0$ the system of equations in (3.8) leads to

$$
N=-3 \frac{1+a}{\beta},
$$

while for $\beta=0$ the solution corresponds to $a=-1$ for all $N$. For a given negative (positive) value of $\beta$ there is a set of positive (negative) values of $a$ which render $N$ integer, the complete set of $(N, a)$ values thus determines the viability of the models. The resulting 


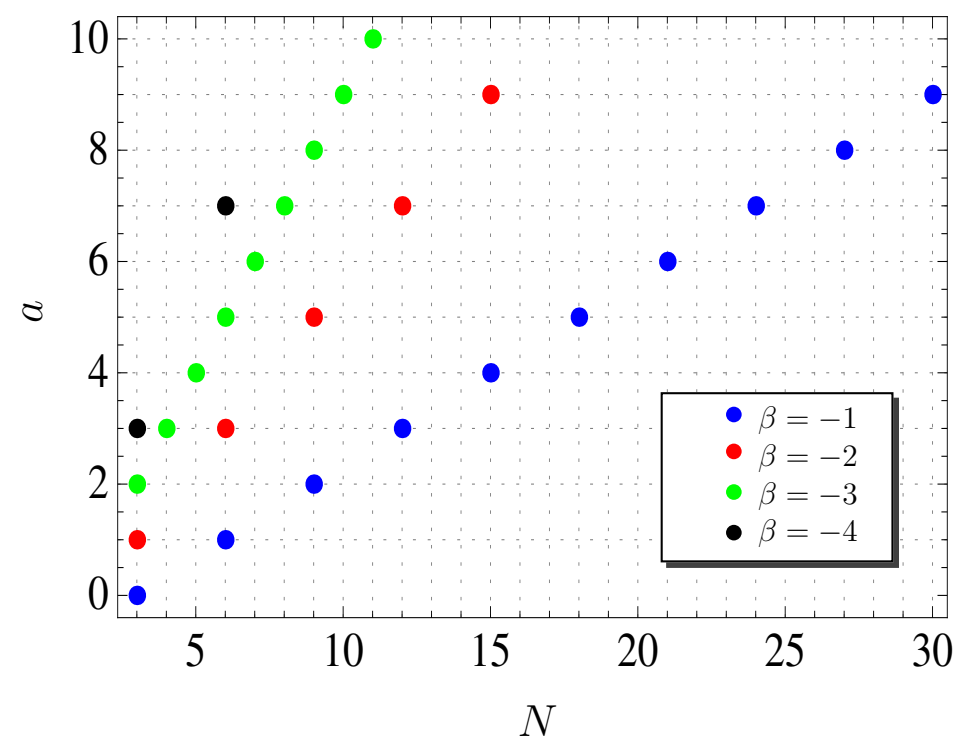

Figure 3. Group order $N$ and possible charge assignments for the charge $a$ which allow the presence of all or part of the dangerous operators. The points in the grid with no colored mark correspond to the viable models. No viable models exist for $N=3$.

sets for values of $\beta=-1,-2,-3,-4$ are displayed in figure 3 . The colored dots indicate those $(N, a)$ choices for which all or some of the dangerous operators will be present, while the remaining pairs correspond to viable $(N, a)$ choices, apart from $N=3$ for which no viable models can be considered no matter the values of the $a$ charge.

\subsection{The type-II seesaw case}

In the absence of further degrees of freedom, the general type-II superpotential involves the following terms ${ }^{3}$

$$
W_{\nu}^{(I I)}=y_{\Delta} L L \Delta_{u}+\lambda_{u} H_{d} H_{d} \Delta_{u}+\lambda_{d} H_{u} H_{u} \Delta_{d}+\mu_{\Delta} \Delta_{u} \Delta_{d}
$$

with $Y\left(\Delta_{u}\right)=+1, Y\left(\Delta_{d}\right)=-1$ and lepton number charges assigned according to $L\left(\Delta_{u}\right)=$ -2 and $L\left(\Delta_{d}\right)=+2$. Since we allow only renormalizable terms, $\xi^{\prime}$ can only be coupled to the last term, in which case $A_{4}$ invariance leads to three possibilities ${ }^{4}$

$$
W_{\nu}^{(I I)} \supset \begin{cases}(\boldsymbol{A}): & \lambda_{\Delta} \Delta_{u}^{\prime} \Delta_{d}^{\prime} \xi^{\prime}: A_{4} \text { non-trivial } \Delta_{u}, \Delta_{d} \\ (\boldsymbol{B}): & \lambda_{\Delta} \Delta_{u}^{\prime \prime} \Delta_{d} \xi^{\prime}: A_{4} \text { non-trivial } \Delta_{u} \\ (\boldsymbol{C}): & \lambda_{\Delta} \Delta_{u} \Delta_{d}^{\prime \prime} \xi^{\prime}: A_{4} \text { non-trivial } \Delta_{d}\end{cases}
$$

Bearing in mind that the new $\Delta L=2$ operator should have $\xi^{\prime}$ appearing twice, general conclusions regarding these possibilities can be drawn:

\footnotetext{
${ }^{3}$ For a contribution to the full neutrino mass matrix a single triplet suffices, however in the supersymmetric case two triplets with opposite hypercharge are needed to avoid the triangle gauge anomaly.

${ }^{4} \Delta_{d}$ must couple to $H_{u}$ so the electroweak triplets can not be $A_{4}$ triplets.
} 

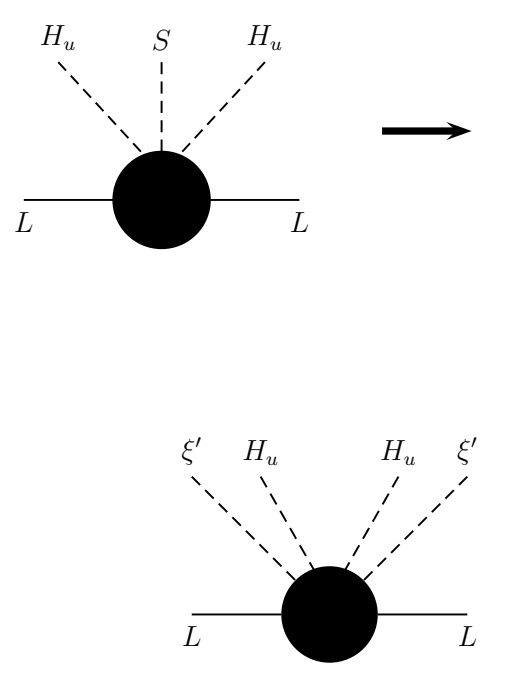

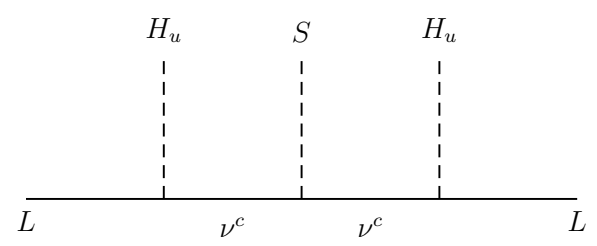

(a)

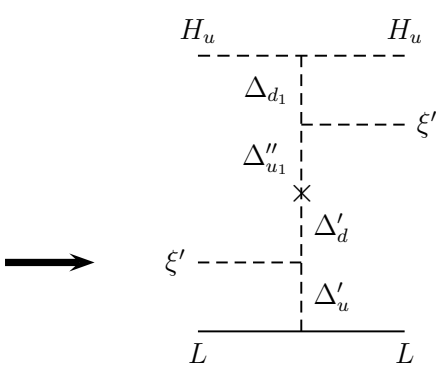

(b)

Figure 4. Lepton-number-breaking effective operators arising from integrating out the heavy typeI and type-II seesaw states, and responsible, after flavor and electroweak symmetry breaking, for the effective neutrino mass matrix with leading TBM-form (Feynman diagrams on top) and small deviations (Feynman diagrams on bottom) accounting for a non-zero reactor mixing angle. For the diagrams on top, $S$ stands for $\xi, \tilde{\xi}$ and $\phi_{\nu}$.

A. Here, the electroweak triplet interactions reduces to:

$$
W_{\nu}^{(I I, A)}=y_{\Delta}\{L L\}^{\prime \prime} \Delta_{u}^{\prime}+\lambda_{\Delta} \Delta_{u}^{\prime} \Delta_{d}^{\prime} \xi^{\prime},
$$

which by itself does not allow the construction of a $\Delta L=2$ operator. Adding a second triplet pair however allows constructing such an operator, with $\Delta_{u_{1}}^{\prime \prime}$ transforms as $\mathbf{1}^{\prime \prime}$, $\Delta_{d_{1}}$ trivially and the coupling $\{L L\}^{\prime} \Delta_{u_{1}}^{\prime \prime}$ absent (otherwise if present will induce a leading order $\{L L\}^{\prime \prime} \xi^{\prime} H_{u} H_{u}$ operator). The latter follows from the following $\mathbb{Z}_{N}$ transformations:

$$
\Delta_{u_{1}}^{\prime \prime} \rightarrow \eta_{N}^{a-1} \Delta_{u_{1}}^{\prime \prime}, \quad \Delta_{d_{1}} \rightarrow \eta_{N}^{-2 a} \Delta_{d_{1}} .
$$

in which case the full superpotential will read

$$
W_{\nu}^{(I I)}=W_{\nu}^{(I I, A)}+\mu_{\Delta_{1}} \Delta_{u_{1}}^{\prime \prime} \Delta_{d}^{\prime}+\lambda_{\Delta_{1}} \Delta_{u_{1}}^{\prime \prime} \Delta_{d_{1}} \xi^{\prime}+\lambda_{d} H_{u} H_{u} \Delta_{d_{1}},
$$

from which then the operator illustrated in figure 4-(b) can be generated. Note also from the $\{L L\}^{\prime \prime} \Delta_{u}^{\prime}$ contraction that this construction leads to a $\Xi^{\prime}$ model.

$\boldsymbol{B}$. In this case the $A_{4}$ transformation properties of the electroweak triplets allow the superpotential to have an extra term, namely

$$
W_{\nu}^{(I I, B)}=y_{\Delta}\{L L\}^{\prime} \Delta_{u}^{\prime \prime}+\lambda_{\Delta} \Delta_{u}^{\prime \prime} \Delta_{d} \xi^{\prime}+\lambda_{d} H_{u} H_{u} \Delta_{d} .
$$

This superpotential allows the construction of a $\Delta L=2$ operator $\{L L\}^{\prime}$ with a single $\xi^{\prime}$, where the charge of $\xi^{\prime}$ would be $+2+a$, leading to the problematic terms in (2.10). 
So, assuring hybridization in this case requires the $\mathbb{Z}_{N}$ symmetry to forbid either the first or the last terms in the superpotential in (3.15) while keeping the remaining. The first term is forbidden provided the triplets satisfy:

$$
\Delta_{d} \rightarrow \eta_{N}^{-2 a} \Delta_{d}, \quad \Delta_{u}^{\prime \prime} \rightarrow \eta_{N}^{a-1} \Delta_{u}^{\prime \prime},
$$

whereas the last term will be absent as long as the following transformations hold

$$
\Delta_{u}^{\prime \prime} \rightarrow \eta_{N}^{-2} \Delta_{u}^{\prime \prime}, \quad \Delta_{d} \rightarrow \eta_{N}^{a-1} \Delta_{d}
$$

We want to generate a $\Delta L=2$ operator with two $\xi^{\prime}$, and in the case where the last term in (3.15) is absent this requires three additional triplet pairs, so we will not consider this possibility in further detail. Instead, on the other option the extra fields enabling the construction of the desirable operator correspond to a pair of triplets transforming as a $A_{4}$ singlets $\mathbf{1}^{\prime}$, i.e. the same field content as the previous case. Indeed, renaming the fields so that the $A_{4}$ representations match the previous nomenclature we conclude that the $\mathbb{Z}_{N}$ charges are unchanged, and thus the superpotential turns out to be given exactly by (3.14), leading to figure 4 -(b) and the same $\Xi^{\prime}$ model.

C. In this case the superpotential involves instead the coupling $H_{d} H_{d} \Delta_{u}$, thus it reads

$$
W_{\nu}^{(I I, C)}=y_{\Delta}\{L L\} \Delta_{u}+\lambda_{\Delta} \Delta_{u} \Delta_{d}^{\prime \prime} \xi^{\prime}+\lambda_{d} H_{d} H_{d} \Delta_{u}
$$

The dimension seven operator can not be constructed, and in contrast to the previous two cases, adding messengers does not fix the problem. This can be readily understood by noting that the requirement of having the $H_{u} H_{u} \Delta_{d}$ coupling calls for the extra scalar triplets involving a "down-type" triplet transforming trivially under $A_{4}$, $\Delta_{d_{1}}$, which in turn requires the presence of a "up-type" triplet, $\Delta_{u_{1}}$, transforming trivially under $A_{4}$ too. The presence of both $\Delta_{u_{1}}$ and $\Delta_{d_{1}}$ lead to new terms which — via the $\mathbb{Z}_{N}$ symmetry - cannot be forbidden simultaneously. Thus, in this case a leading order dimension five operator will be unavoidable. It might be that by allowing extra triplet pairs the dimension seven operator can be constructed in the absence of a dimension five operator, here however we will not add further comments on this possibility.

The full models can then be outlined as follows: the type-I seesaw sector corresponds to a UV complete AF-inspired model as in [35] and so at the LO produces a TBM flavor structure (without additional NLO contributions due to the specific UV completion). The sector dictating the hybridization is a type-II seesaw sector with a flavor structure driven by the same $A_{4}$ symmetry, with a non-trivial flavon singlet responsible for the deviations, that only couples through the electroweak triplets. Although at the renormalizable level the singlet can be coupled to the electroweak triplets in three different manners, only two choices allow for minimality (in the sense of the number of triplet pairs) and they both lead to the same model. Determined by the hybridization statement, minimality requires the type-II sector to involve two triplet pairs. Table 4 shows the transformation properties 


\begin{tabular}{|c|c|cccc|cc|}
\hline Fields & $\nu^{c}$ & $\Delta_{u}^{\prime}$ & $\Delta_{d}^{\prime}$ & $\Delta_{u_{1}}^{\prime \prime}$ & $\Delta_{d_{1}}$ & $\xi^{\prime}$ & $\xi^{0^{\prime}}$ \\
\hline$A_{4}$ & $\mathbf{3}$ & $\mathbf{1}^{\prime}$ & $\mathbf{1}^{\prime}$ & $\mathbf{1}^{\prime \prime}$ & $\mathbf{1}$ & $\mathbf{1}^{\prime}$ & $\mathbf{1}^{\prime}$ \\
$\mathbb{Z}_{N}$ & $-1-a$ & -2 & $1-a$ & $-1+a$ & $-2 a$ & $1+a$ & $-2(1+a)$ \\
$\mathrm{U}(1)_{\mathrm{FN}}$ & 0 & 0 & 0 & 0 & 0 & 0 & 0 \\
$\mathrm{U}(1)_{R}$ & 0 & 0 & 2 & 0 & 2 & 0 & 2 \\
$\mathrm{U}(1)_{Y}$ & 0 & +1 & -1 & +1 & -1 & 0 & 0 \\
\hline
\end{tabular}

Table 4. Transformation properties of the messenger fields in the type-I and type-II sectors of the $A_{4}$-based hybrid model. The last two columns correspond to the flavon fields needed in the construction of an $A_{4}$-based hybrid model.

of the different messenger fields involved in the minimal $A_{4}$-based hybrid $\Xi^{\prime}$ model with type-I and type-II seesaw. The $\Xi^{\prime \prime}$ model can be obtained by swapping $\xi^{\prime}, \xi^{0^{\prime}}$ with $\xi^{\prime \prime}, \xi^{0^{\prime \prime}}$ and also adjusting the messengers accordingly.

We finally come to the issue of the singlet $\xi^{\prime}$ vev. Once getting rid of the dangerous terms in (3.4) the new "driving" sector superpotential reduces to:

$$
W_{\phi}^{\mathrm{New}}=\bar{g}_{4} \xi^{0^{\prime}}\left\{\phi_{l} \phi_{\nu}\right\}^{\prime \prime}+\bar{g}_{5} \xi^{0^{\prime}} \xi^{\prime} \xi^{\prime}
$$

from which the minimization condition $\partial W_{\phi}^{\text {New }} / \partial \xi^{0^{\prime}}=0$ allows the determination of the $\xi^{\prime}$ vev, namely

$$
\left\langle\xi^{\prime}\right\rangle \equiv \bar{u}=\sqrt{-\frac{\bar{g}_{4} v_{l} v_{\nu}}{\bar{g}_{5}}}
$$

\subsection{The type-III seesaw case}

We can replace type-II seesaw with type-III seesaw to obtain different UV completions. As it turns out, within this case there is a minimal model requiring two additional superfields (compared to four in section 3.1). We add superfields $T_{0}$ and $T_{1}$ :

$$
W_{\nu}^{(I I I)}=y_{T}\left\{L T_{1}\right\} H_{u}+\left\{T_{1} T_{1}\right\} \xi+\left\{\phi_{\nu} T_{1} T_{1}\right\}+\lambda_{T}\left\{T_{1} T_{0}\right\}^{\prime \prime} \xi^{\prime}+\mu_{T}\left\{T_{0} T_{0}\right\},
$$

The assignments are quite distinct from the type-II case: here the messengers are $A_{4}$ triplets with R-charge 1 like $\nu^{c}$, as shown in see table 5. As $T_{1}$ and $\nu^{c}$ share all the assignments (apart from $\mathrm{SU}(2)$ ), the terms $y_{T}\left\{L T_{1}\right\} H_{u},\left\{T_{1} T_{1}\right\} \xi$ and $\left\{\phi_{\nu} T_{1} T_{1}\right\}$ appear. The eigenvectors [7] of mass matrix generated by these operators alone are of TBM-form, so even though they arise from type-III they can be absorbed into $A$ and $B$ in (2.7). It is the presence of $T_{0}$ that distinguishes type-III from type-I. The vev of $\xi^{\prime}$ can be obtained through (3.20) as in section 3.1. The diagram associated with the deviations is illustrated in figure 5 . Checking the $A_{4}$ triplet indices through diagram one can correctly identify that this particular UV completion corresponds to a $\{L L\}^{\prime \prime}$ contraction, i.e. a $\Xi^{\prime}$ model (the $\Xi^{\prime \prime}$ model corresponds to swapping $\xi^{\prime}, \xi^{0^{\prime}}$ with $\left.\xi^{\prime \prime}, \xi^{0^{\prime \prime}}\right)$. 


\begin{tabular}{|c|c|cc|cc|}
\hline Fields & $\nu^{c}$ & $T_{1}$ & $T_{0}$ & $\xi^{\prime}$ & $\xi^{0^{\prime}}$ \\
\hline$A_{4}$ & $\mathbf{3}$ & $\mathbf{3}$ & $\mathbf{3}$ & $\mathbf{1}^{\prime}$ & $\mathbf{1}^{\prime}$ \\
$\mathbb{Z}_{N}$ & $-1-a$ & $-1-a$ & 0 & $1+a$ & $-2(1+a)$ \\
$\mathrm{U}(1)_{\mathrm{FN}}$ & 0 & 0 & 0 & 0 & 0 \\
$\mathrm{U}(1)_{R}$ & 1 & 1 & 1 & 0 & 2 \\
$\mathrm{U}(1)_{Y}$ & 0 & 0 & 0 & 0 & 0 \\
\hline
\end{tabular}

Table 5. Transformation properties of the messenger fields in the type-I and type-III sectors of the $A_{4}$-based hybrid model.
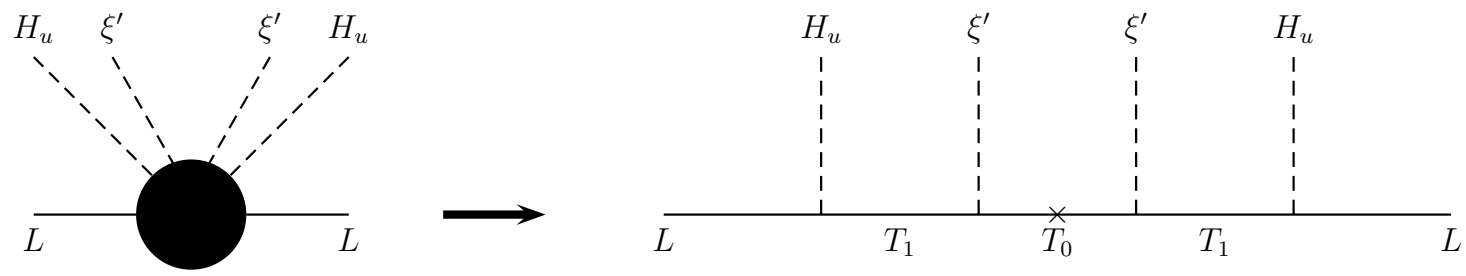

Figure 5. Lepton-number-breaking effective operator arising from integrating out the heavy typeIII seesaw states.

\section{Conclusions}

Regardless of the mechanism responsible for neutrino masses, the neutrino mass matrix should have a leading TBM-form with small deviations accounting for a non-vanishing reactor mixing angle, as can be proved - in a model-independent fashion — with the aid of the eigenvector decomposition of the neutrino mass matrix combined with current neutrino oscillation data [7]. Although such structure can emerge in a variety of ways, here we take the position that it suggests different mechanisms are participating in the generation of neutrino masses, i.e. that we have "hybrid neutrino masses".

We have studied the feasibility of this "interpretation" under rather general conditions, without specifying the mechanisms at work. Our analysis then progressed by assuming an underlying $A_{4}$ flavor symmetry in the lepton sector, and through $\Delta L=2 \mathrm{LO}$ effective operators. Enforcing one of these operators to generate a TBM-form structure, we investigated the minimal $A_{4}$ representations capable of producing deviations from TBM in agreement with data. After identifying these representations we have proved that independently of the mechanisms assumed, fixing the reactor angle in its experimental range always yield solar and atmospheric mixing angles consistent with data. In this sense, deviations from TBM induced by "hybrid neutrino masses" alleviate the problem found in typical $A_{4}$ models, where NLO effective operators producing a non-zero reactor angle quite often lead to values for the solar and atmospheric angles which are inconsistent with data [12-14].

In order to illustrate this picture, we have constructed an UV completion based on an interplay between type-I and either type-II or type-III seesaw. Still under the assumption of an $A_{4}$ flavor symmetry, the type-I seesaw contribution has been taken as responsible for the leading TBM-form, while the deviations are driven by the other contribution. 


\section{Acknowledgments}

DAS wants to thanks Avelino Vicente for useful conversations, also NORDITA for hospitality during the completion of this work. DAS is supported by a "Chargé de Recherches" contract funded by the Belgian FNRS agency. The work of IdMV is supported by the Swiss National Science Foundation.

Open Access. This article is distributed under the terms of the Creative Commons Attribution License (CC-BY 4.0), which permits any use, distribution and reproduction in any medium, provided the original author(s) and source are credited.

\section{References}

[1] P.F. Harrison, D.H. Perkins and W.G. Scott, Tri-bimaximal mixing and the neutrino oscillation data, Phys. Lett. B 530 (2002) 167 [hep-ph/0202074] [INSPIRE].

[2] DAYA-BAY collaboration, F.P. An et al., Observation of electron-antineutrino disappearance at Daya Bay, Phys. Rev. Lett. 108 (2012) 171803 [arXiv:1203.1669] [INSPIRE].

[3] RENO collaboration, J.K. Ahn et al., Observation of Reactor Electron Antineutrino Disappearance in the RENO Experiment, Phys. Rev. Lett. 108 (2012) 191802 [arXiv: 1204.0626] [INSPIRE].

[4] D.V. Forero, M. Tortola and J.W.F. Valle, Global status of neutrino oscillation parameters after Neutrino-2012, Phys. Rev. D 86 (2012) 073012 [arXiv: 1205.4018] [INSPIRE].

[5] M.C. Gonzalez-Garcia, M. Maltoni, J. Salvado and T. Schwetz, Global fit to three neutrino mixing: critical look at present precision, JHEP 12 (2012) 123 [arXiv:1209.3023] [INSPIRE].

[6] G.L. Fogli, E. Lisi, A. Marrone, D. Montanino, A. Palazzo et al., Global analysis of neutrino masses, mixings and phases: entering the era of leptonic CP-violation searches, Phys. Rev. D 86 (2012) 013012 [arXiv:1205.5254] [INSPIRE].

[7] D. Aristizabal Sierra, I. de Medeiros Varzielas and E. Houet, Eigenvector-based approach to neutrino mixing, Phys. Rev. D 87 (2013) 093009 [arXiv: 1302.6499] [INSPIRE].

[8] D. Aristizabal Sierra, F. Bazzocchi and I. de Medeiros Varzielas, Leptogenesis in flavor models with type-I and II seesaws, Nucl. Phys. B 858 (2012) 196 [arXiv:1112.1843] [INSPIRE].

[9] E. Bertuzzo, P. Di Bari, F. Feruglio and E. Nardi, Flavor symmetries, leptogenesis and the absolute neutrino mass scale, JHEP 11 (2009) 036 [arXiv:0908.0161] [INSPIRE].

[10] D. Aristizabal Sierra, F. Bazzocchi, I. de Medeiros Varzielas, L. Merlo and S. Morisi, Tri-Bimaximal Lepton Mixing and Leptogenesis, Nucl. Phys. B 827 (2010) 34 [arXiv: 0908.0907] [INSPIRE].

[11] R.G. Felipe and H. Serodio, Constraints on leptogenesis from a symmetry viewpoint, Phys. Rev. D 81 (2010) 053008 [arXiv:0908.2947] [INSPIRE].

[12] Y. Lin, Tri-bimaximal Neutrino Mixing from $A_{4}$ and $\theta_{13} \sim \theta_{C}$, Nucl. Phys. B 824 (2010) 95 [arXiv:0905.3534] [INSPIRE].

[13] G. Altarelli, F. Feruglio, L. Merlo and E. Stamou, Discrete Flavour Groups, $\theta_{13}$ and Lepton Flavour Violation, JHEP 08 (2012) 021 [arXiv:1205.4670] [INSPIRE]. 
[14] I. de Medeiros Varzielas and D. Pidt, UV completions of flavour models and large $\theta_{13}$, JHEP 03 (2013) 065 [arXiv: 1211.5370] [INSPIRE].

[15] W. Rodejohann, Type II seesaw mechanism, deviations from bimaximal neutrino mixing and leptogenesis, Phys. Rev. D 70 (2004) 073010 [hep-ph/0403236] [InSPIRE].

[16] M. Lindner and W. Rodejohann, Large and almost maximal neutrino mixing within the type-II see-saw mechanism, JHEP 05 (2007) 089 [hep-ph/0703171] [INSPIRE].

[17] A. Adulpravitchai and R. Takahashi, $A_{4}$ Flavor Models in Split Seesaw Mechanism, JHEP 09 (2011) 127 [arXiv: 1107.3829] [INSPIRE].

[18] D. Borah, Deviations from Tri-Bimaximal Neutrino Mixing Using Type II Seesaw, Nucl. Phys. B 876 (2013) 575 [arXiv: 1307.2426] [INSPIRE].

[19] D. Borah, S. Patra and P. Pritimita, Sub-dominant type-II seesaw as an origin of non-zero $\theta_{13}$ in $\mathrm{SO}(10)$ model with TeV scale Z' gauge boson, Nucl. Phys. B 881 (2014) 444 [arXiv: 1312.5885] [INSPIRE].

[20] D. Borah, Type II Seesaw Origin of Non-zero $\theta_{13}, \delta_{C P}$ and Leptogenesis, arXiv: 1403.7636 [INSPIRE].

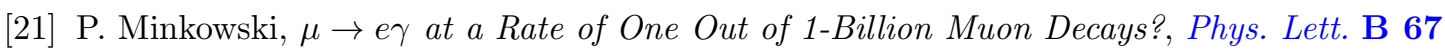
(1977) 421 [INSPIRE].

[22] T. Yanagida, in Proc. of Workshop on Unified Theory and Baryon number in the Universe, O. Sawada and A. Sugamoto eds., KEK, Tsukuba (1979).

[23] M. Gell-Mann, P. Ramond and R. Slansky, Complex Spinors and Unified Theories, Conf. Proc. C 790927 (1979) 315 [arXiv:1306.4669] [InSPIRE].

[24] P. Ramond, The Family Group in Grand Unified Theories, hep-ph/9809459 [INSPIRE].

[25] S.L. Glashow, in Quarks and Leptons, Cargèse lectures, M. Lévy ed., Plenum, New York (1980).

[26] R.N. Mohapatra and G. Senjanović, Neutrino Mass and Spontaneous Parity Violation, Phys. Rev. Lett. 44 (1980) 912 [INSPIRE].

[27] J. Schechter and J.W.F. Valle, Neutrino Masses in $\mathrm{SU}(2) \times \mathrm{U}(1)$ Theories, Phys. Rev. D 22 (1980) 2227 [INSPIRE].

[28] J. Schechter and J.W.F. Valle, Neutrino Decay and Spontaneous Violation of Lepton Number, Phys. Rev. D 25 (1982) 774 [INSPIRE].

[29] J. Schechter and J.W.F. Valle, Neutrino Masses in $\mathrm{SU}(2) \times \mathrm{U}(1)$ Theories, Phys. Rev. D 22 (1980) 2227 [INSPIRE].

[30] G. Lazarides, Q. Shafi and C. Wetterich, Proton Lifetime and Fermion Masses in an SO(10) Model, Nucl. Phys. B 181 (1981) 287 [InSPIRE].

[31] R.N. Mohapatra and G. Senjanović, Neutrino Masses and Mixings in Gauge Models with Spontaneous Parity Violation, Phys. Rev. D 23 (1981) 165 [INSPIRE].

[32] C. Wetterich, Neutrino Masses and the Scale of B-L Violation, Nucl. Phys. B 187 (1981) 343 [INSPIRE].

[33] G. Altarelli and F. Feruglio, Tri-bimaximal neutrino mixing, $A_{4}$ and the modular symmetry, Nucl. Phys. B 741 (2006) 215 [hep-ph/0512103] [INSPIRE]. 
[34] C.D. Froggatt and H.B. Nielsen, Hierarchy of Quark Masses, Cabibbo Angles and CP-violation, Nucl. Phys. B 147 (1979) 277 [INSPIRE].

[35] I. de Medeiros Varzielas and L. Merlo, Ultraviolet Completion of Flavour Models, JHEP 02 (2011) 062 [arXiv:1011.6662] [INSPIRE].

[36] R. Foot, H. Lew, X.G. He and G.C. Joshi, Seesaw Neutrino Masses Induced by a Triplet of Leptons, Z. Phys. C 44 (1989) 441 [inSPIRE].

[37] B. Brahmachari, S. Choubey and M. Mitra, The $A_{4}$ flavor symmetry and neutrino phenomenology, Phys. Rev. D 77 (2008) 073008 [Erratum ibid. D 77 (2008) 119901] [arXiv: 0801.3554] [INSPIRE].

[38] J. Barry and W. Rodejohann, Deviations from tribimaximal mixing due to the vacuum expectation value misalignment in $A_{4}$ models, Phys. Rev. D 81 (2010) 093002 [Erratum ibid. D 81 (2010) 119901] [arXiv: 1003.2385] [INSPIRE].

[39] Y. Shimizu, M. Tanimoto and A. Watanabe, Breaking Tri-bimaximal Mixing and Large $\theta_{13}$, Prog. Theor. Phys. 126 (2011) 81 [arXiv:1105.2929] [INSPIRE].

[40] J. Barry and W. Rodejohann, Neutrino Mass Sum-rules in Flavor Symmetry Models, Nucl. Phys. B 842 (2011) 33 [arXiv: 1007.5217] [INSPIRE]. 